\title{
Asymptotic results for tail probabilities of sums of dependent heavy-tailed random variables
}

\author{
${ }^{a}$ Kam Chuen Yuen, and ${ }^{b}$ Chuancun Yin \\ ${ }^{a}$ Department of Statistics and Actuarial Science, The University of Hong Kong, \\ Pokfulam Road, Hong Kong \\ E-mail: kcyuen@hku.hk \\ ${ }^{b}$ School of Mathematical Sciences, Qufu Normal University \\ Shandong 273165, P.R. China \\ E-mail: ccyin@mail.qfnu.edu.cn
}

\begin{abstract}
Let $\left\{X_{1}, X_{2}, \cdots\right\}$ be a sequence of dependent heavy-tailed random variables with distributions $F_{1}, F_{2}, \cdots$ on $(-\infty, \infty)$, and let $\tau$ be a nonnegative integer-valued random variable independent of the sequence $\left\{X_{k}, k \geq 1\right\}$. In this framework, we study the asymptotic behavior of the tail probabilities of the quantities $X_{(n)}=\max _{1 \leq k \leq n} X_{k}$, $S_{n}=\sum_{k=1}^{n} X_{k}$ and $S_{(n)}=\max _{1 \leq k \leq n} S_{k}$ for $n>1$, and for those of their randomized versions $X_{(\tau)}, S_{\tau}$ and $S_{(\tau)}$. We also consider applications of the results obtained to some commonly-used risk processes.
\end{abstract}

Keywords Asymptotic independence; asymptotic tail probability; copula; heavy-tailed distributions; partial sum; risk process

Mathematics Subject Classification (2000): Primary 62E20; Secondary 91B30; 62P05 


\section{Introduction}

Throughout this paper, let $\left\{X_{1}, X_{2}, \cdots\right\}$ be a sequence of random variables with distributions $F_{1}, F_{2}, \cdots$ supported on $\mathbb{R}:=(-\infty, \infty)$ satisfying $\bar{F}_{k}(x)=1-F_{k}(x)>0$ for all $x$. For $n \geq 1$, we write

$$
X_{(n)}=\max _{1 \leq k \leq n} X_{k}, \quad S_{n}=\sum_{k=1}^{n} X_{k}, \quad S_{(n)}=\max _{1 \leq k \leq n} S_{k} .
$$

Let $\tau$ be a counting random variable independent of $\left\{X_{k}, k \geq 1\right\}$. Then, the randomized versions of $X_{(n)}, S_{n}, S_{(n)}$ are given by $X_{(\tau)}, S_{\tau}, S_{(\tau)}$. Tail probabilities of the quantities $X_{(n)}, S_{n}, S_{(n)}, X_{(\tau)}, S_{\tau}, S_{(\tau)}$ with heavy-tailed random variables are of great interest in finance, insurance and many other disciplines. Since accurate distributions for these quantities are not available in most cases, the study of asymptotic relationships for their tail probabilities becomes important. Many results have been derived under different degrees of generality in the literature. In particular, most of the results are for independent $X_{1}, \cdots, X_{n}$ with distributions belonging to the class of subexponential distributions.

For two independent random variables $X$ and $Y$ with distribution functions $F$ and $G$ supported on $(-\infty, \infty)$, we denote by $F * G(x)=\int_{-\infty}^{\infty} F(x-y) d G(y),-\infty<x<\infty$, the convolution of $F$ and $G$, and by $F^{* n}=F * F \cdots * F$ the $n$-fold convolution of $F$. Unless otherwise stated, all limit relations are for $x \rightarrow \infty$. By definition, a distribution $F$ on $[0, \infty)$ is said to be subexponential $(F \in \mathcal{S})$ if the relation $\overline{F^{* 2}}(x) \sim 2 \bar{F}(x)(x \rightarrow$ $\infty)$ holds where the symbol $\sim$ means that the ratio of the two sides tends to 1 . More generally, a distribution function $F$ on $(-\infty, \infty)$ belongs to the subexponential class $\mathcal{S}$ if $F^{+}(x)=F(x) 1(x \geq 0)$ does, where $1(\cdot)$ is the indicator function. A recent account on tail asymptotic results for the sum of two independent random variables can be found in Foss and Korshunov [11]. They proved that, for two distributions $F_{1}$ and $F_{2}$ on $[0, \infty)$, if one of $F_{1}$ and $F_{2}$ is heavy tailed (see the definition below), then

$$
\liminf _{x \rightarrow \infty} \frac{\overline{F_{1} * F_{2}}(x)}{\overline{F_{1}}(x)+\overline{F_{2}}(x)}=1,
$$

and that, for any heavy-tailed distribution $F$,

$$
\liminf _{x \rightarrow \infty} \frac{\overline{F^{* 2}}(x)}{\bar{F}(x)}=2 .
$$


Denisov et al. [6] extended (1.2) to

$$
\liminf _{x \rightarrow \infty} \frac{\overline{F^{* \tau}}(x)}{\bar{F}(x)}=E \tau,
$$

with $\tau$ being a light-tailed random variable. Furthermore, for any heavy-tailed distribution $F$ on $\mathbb{R}^{+}$with finite mean, Denisov et al. [7] showed that if $P(c \tau>x)=o(\bar{F}(x))$ for some $c>E X$ as $x \rightarrow \infty$, then (1.3) holds. Also, if $F$ is subexponential and $\tau$ is light tailed and independent of the summands, then

$$
P\left(S_{\tau}>x\right) \sim \bar{F}(x) E \tau, x \rightarrow \infty
$$

Note that all the above-mentioned results were established for independent nonnegative random variables. When the random variables are possibly negative and dependent according to certain structures, the validity of these results remains to be studied.

We now consider three examples in which some of the above relations do not hold. The first comes from Yu et al. [25] while the last two are extracted from Albrecher et al. $[1]$.

Example 1.1. Assume that $X$ is a discrete random variable with masses $p_{n}=(X=$ $\left.2^{n+1}-1\right)=2^{-n-1}, n \geq 0$. Denote its distribution by $\rho$. Then, for any $0<q<1$, define $F=q \rho+(1-q) \sigma$, where $\sigma$ is a non-degenerated distribution on a subset of $(-\infty, 0]$. Without loss of generality, we assume that $\sigma$ has support on $[-3,0)$, and that $\sigma(-2)-\sigma(-3)=\delta>0$. Then, $F$ is heavy-tailed but does not belong to the class $\mathcal{L}$ of distributions with long tails $(F \notin \mathcal{L})$. Also, it satisfies

$$
\liminf _{x \rightarrow \infty} \frac{\overline{F^{* 2}}(x)}{\bar{F}(x)}<2 .
$$

Example 1.2. Let $X_{1}$ and $X_{2}$ have marginal distribution function $F$ belonging to the subexponential class $\mathcal{S}$. Then, there exists a copula for $X_{1}$ and $X_{2}$ such that

$$
\lim _{x \rightarrow \infty} \frac{P\left(X_{1}+X_{2}>x\right)}{P\left(X_{1}>x\right)}=\infty .
$$

Example 1.3. Assume that random variables $X_{1}$ and $X_{2}$ are comonotone dependent with common distribution $F=1-\bar{F}$ where $\bar{F} \in \mathcal{R}_{-\alpha}$ is regularly varying at infinity with some index $\alpha>0$. Then, we have

$$
\lim _{x \rightarrow \infty} \frac{P\left(X_{1}+X_{2}>x\right)}{P\left(X_{1}>x\right)}=2^{\alpha} .
$$


These examples indicate that relations (1.1)-(1.4) may not hold for heavy-tailed distributions supported on $[a, \infty)$ with $a<0$ or for dependent heavy-tailed distributions. More examples can be found in Albrecher et al. [1].

The purpose of this paper is to find sufficient conditions under which relations (1.1)(1.4) hold for possibly negative, non-identically distributed, and dependent heavy-tailed random variables. The outline of the paper is as follows. Section 2 presents several classes of heavy-tailed distributions and the dependence assumptions used in later sections. Section 3 is devoted to the tail behavior of $X_{(n)}, S_{n}$ and $S_{(n)}$. Section 4 investigates the tail behavior of $X_{(\tau)}, S_{\tau}$ and $S_{(\tau)}$. Section 5 presents applications of the main results to risk theory.

\section{Preliminaries}

A random variable $X$ (or its distribution $F$ ) is heavy tailed (to the right) if $E \exp (\alpha X)=$ $\infty$ for all $\alpha>0$, and light tailed otherwise. One of the most important classes of heavytailed distributions is the subexponential class. Closely related to the class $\mathcal{S}$ are the class $\mathcal{S}^{*}$, the class $\mathcal{D}$ of distributions with dominatedly varying tails, and the class $\mathcal{L}$ of distributions with long tails. By definition, a distribution function $F$ on $\mathbb{R}$ with finite mean belongs to the class $\mathcal{S}^{*}$ if and only if $\bar{F}(x)>0$ for all $x$ and $\int_{0}^{x} \bar{F}(x-y) \bar{F}(y) d y \sim 2 m_{F^{+}} \bar{F}(x)$, as $x \rightarrow \infty$, where $m_{F^{+}}=\int_{0}^{\infty} \bar{F}(x) d x$ is the mean of $F^{+}$. It is known that if $F \in \mathcal{S}^{*}$, then both $F$ and $F_{I}$ are subexponential, where $F_{I}$ is defined by $\overline{F_{I}}(x)=\min \left(1, \int_{x}^{\infty} \bar{F}(t) d t\right)$; see Klüppelberg [15]. A distribution function $F$ with support on $(-\infty, \infty)$ belongs to the class $\mathcal{D}$ if $\lim _{\sup _{x \rightarrow \infty}} \bar{F}(x y) / \bar{F}(x)<\infty$ holds for some (or equivalently for all) $0<y<1$. Obviously, if $F \in \mathcal{D}$, then, for any $y>0, \bar{F}(x y)$ and $\bar{F}(x)$ are of the same order as $x \rightarrow \infty$ in the sense that

$$
0<\liminf _{x \rightarrow \infty} \frac{\bar{F}(x y)}{\bar{F}(x)} \leq \limsup _{x \rightarrow \infty} \frac{\bar{F}(x y)}{\bar{F}(x)}<\infty .
$$

A distribution function $F$ is said to belong to the class $\mathcal{L}$ if $\lim _{x \rightarrow \infty} \bar{F}(x+y) / \bar{F}(x)=1$ holds for some (or equivalently for all) $y$. One can easily check that for a distribution $F \in \mathcal{L}$, there exists a positive function $h(x) \rightarrow \infty$ such that $\bar{F}(x+h(x)) \sim \bar{F}(x)$. The class $\mathcal{S}^{*}$ and the intersection $\mathcal{D} \cap \mathcal{L}$ are two well-known subclasses of subexponential distribution functions. For details of these classes of heavy-tailed distributions and their 
applications, the reader is referred to Asmussen [2], Bingham et al. [3], Embrechts et al. [9], and Embrechts et al. [10]. Furthermore, a distribution $F$ is said to be strongly subexponential, denoted by $F \in \mathcal{S}_{*}$, if $\overline{F_{h}^{* 2}}(x) \sim 2 \overline{F_{h}}(x)$, uniformly in $h \in[1, \infty)$, where the distribution $F_{h}$ is defined as

$$
F_{h}(x)=\min \left(1, \int_{x}^{x+h} F(t) d t\right), \quad x>0 .
$$

See Korshunov [17] for sufficient conditions for some distribution to belong to the class $\mathcal{S}_{*}$. Kaas and Tang [14] proved that $\mathcal{S}_{*}$ is a subclass of $\mathcal{S}$ while Denisov et al. [5] showed that $\mathcal{S}^{*}$ is a subclass of $\mathcal{S}_{*}$. The relations between the above-mentioned classes are summarized as follows: $\mathcal{D} \cap \mathcal{L} \subset \mathcal{S}_{*} \subset \mathcal{S} \subset \mathcal{K}$, and that, if the distribution function $F$ has a finite mean, then $F \in \mathcal{D} \cap \mathcal{L} \Rightarrow F \in \mathcal{S}^{*} \subset \mathcal{S}_{*}$, where $\mathcal{K}$ represents the class of distribution functions with heavy tails.

Recall that $X_{1}, \cdots, X_{n}$ are $n$ real-valued random variables with distributions $F_{1}, \cdots, F_{n}$, respectively. Here, we assume that these random variables are dependent. To model the dependence of a multivariate distribution with non-identical marginals, one may use the theory of copulas (see, for example, Nelsen [19]). A copula is a multivariate joint distribution defined on the $n$-dimensional unit cube $[0,1]^{n}$ such that every marginal distribution is uniform on the interval $[0,1]$. By Sklar's theorem, for a multivariate joint distribution $F$ of a random vector $\left(X_{1}, \cdots, X_{n}\right)$ with marginals $F_{1}, \cdots, F_{n}$, there exists a copula $C$ such that

$$
F\left(x_{1}, \cdots, x_{n}\right)=C\left(F_{1}\left(x_{1}\right), \cdots, F_{n}\left(x_{n}\right)\right), \quad\left(x_{1}, \cdots, x_{n}\right) \in \mathbb{R}^{n} .
$$

If $F_{1}, \cdots, F_{n}$ are all continuous, then $C$ is unique and can be written as

$$
C\left(u_{1}, \cdots, u_{n}\right)=P\left(F_{1}\left(X_{1}\right) \leq u_{1}, \cdots, F_{n}\left(X_{n}\right) \leq u_{n}\right)=F\left(F_{1}^{-1}\left(u_{1}\right), \cdots, F_{n}^{-1}\left(u_{n}\right)\right),
$$

for any $\left(u_{1}, \cdots, u_{n}\right) \in[0,1]^{n}$. Conversely, if $C$ is a copula and $F_{1}, \cdots, F_{n}$ are distribution functions, then $F$ defined in (2.2) is a multivariate joint distribution with marginals $F_{1}, \cdots, F_{n}$.

For notional convenience, we state the following four assumptions regarding the random variables $X_{1}, \cdots, X_{n}$.

H1. Assume that $X_{1}, \cdots, X_{n}$ satisfy

$$
\lim _{x \rightarrow \infty} \frac{P\left(X_{i}>x, X_{j}>x\right)}{\overline{F_{i}}(x)+\overline{F_{j}}(x)}=0
$$


for all $1 \leq i \neq j \leq n$.

This dependence assumption was first introduced in Chen and Yuen [4] and $\left\{X_{i}\right\}$ is called pairwise quasi-asymptotically independence.

H2. Assume that

$$
\hat{\lambda}_{i j}=\lim _{x_{i} \wedge x_{j} \rightarrow \infty} P\left(\left|X_{i}\right|>x_{i} \mid X_{j}>x_{j}\right)=0 .
$$

holds for all $1 \leq i \neq j \leq n$, where $x_{i} \wedge x_{j}=\min \left(x_{i}, x_{j}\right)$. This concept is related to the socalled asymptotic independence; for example, see Resnick [23]. Note that the asymptotic independence means a large value in one component is unlikely to be accompanied by a large value in another.

H3. Assume that there exist positive constants $x_{0}$ and $c_{0}$ such that the inequality

$$
P\left(X_{i}>x_{i} \mid X_{j}=x_{j}, j \in J\right) \leq c_{0} \overline{F_{i}}\left(x_{i}\right)
$$

holds for all $1 \leq i \leq n, \emptyset \neq J \subset\{1,2, \cdots, n\} \backslash\{i\}, x_{i}>x_{0}$, and $x_{j}>x_{0}$ with $j \in J$. When $x_{j}$ is not a possible value of $X_{j}$, the conditional probability above is simply understood as 0. Note that this dependence assumption was used in Geluk and Tang [14].

H4. Let $X_{1}, \cdots, X_{n}$ be dependent. Assume that the dependent structure is governed by an absolutely continuous copula $C\left(u_{1}, \cdots, u_{n}\right)$ such that there exists positive constant $M<\infty$ with $c\left(u_{1}, \cdots, u_{n}\right) \leq M$ for all $\left(u_{1}, \cdots, u_{n}\right) \in[0,1]^{n}$, where $c$ is the copula density given by

$$
c\left(u_{1}, \cdots, u_{n}\right)=\frac{\partial^{n} C\left(u_{1}, \cdots, u_{n}\right)}{\partial u_{1} \cdots \partial u_{n}} .
$$

Remark 2.1. It is obvious that $\mathbf{H} \mathbf{2}$ implies H1. Also, we see in Geluk and Tang [13] that $\mathbf{H 3}$ implies $\mathbf{H 2}$. If $J$ is the set of a single point, then one can show that $\mathbf{H} \mathbf{4}$ implies H3. In fact, let $\left(X_{1}^{*}, \cdots, X_{n}^{*}\right)$ be an independent copy of $\left(X_{1}, \cdots, X_{n}\right)$, then

$$
\begin{aligned}
P\left(X_{i}>x_{i} \mid X_{j}=x_{j}, j \in J\right) & =\lim _{\Delta x_{j} \rightarrow 0} P\left(X_{i}>x_{i} \mid x_{j} \leq X_{j}<x_{j}+\Delta x_{j}, j \in J\right) \\
& =\lim _{\Delta x_{j} \rightarrow 0} \frac{P\left(X_{i}>x_{i}, x_{j} \leq X_{j}<x_{j}+\Delta x_{j}, j \in J\right)}{P\left(x_{j} \leq X_{j}<x_{j}+\Delta x_{j}, j \in J\right)} \\
& \leq M \lim _{\Delta x_{j} \rightarrow 0} \frac{P\left(X_{i}^{*}>x_{i}, x_{j} \leq X_{j}^{*}<x_{j}+\Delta x_{j}, j \in J\right)}{P\left(x_{j} \leq X_{j}<x_{j}+\Delta x_{j}, j \in J\right)} \\
& \leq M P\left(X_{i}^{*}>x_{i}\right),
\end{aligned}
$$


as desired. Note that from the proof above, it is easy to see that if additionally $c$ satisfies $c\left(u_{1}, \cdots, u_{n}\right) \geq m$ for all $\left(u_{1}, \cdots, u_{n}\right) \in[0,1]^{n}$, where $m$ is a positive constant, then $\mathbf{H} 4$ implies H3.

To end the section, we present an example in which the four assumptions are satisfied.

Example 2.1. A joint $n$-dimensional distribution is called a Farlie-Gumbel-Morgenstern (FGM) distribution if it has the form

$$
\left.F\left(x_{1}, \cdots, x_{n}\right)=C\left(F_{1}\left(x_{1}\right), \cdots, F_{n}\left(x_{n}\right)\right)\right), \quad\left(x_{1}, \cdots, x_{n}\right) \in \mathbb{R}^{n},
$$

where $F_{1}, \cdots, F_{n}$ are the one-dimensional marginals, and the copula $C$ is given by

$$
C\left(u_{1}, \cdots, u_{n}\right)=\prod_{k=1}^{n} u_{k}\left(1+\sum_{1 \leq i<j \leq n} a_{i j}\left(1-u_{i}\right)\left(1-u_{j}\right)\right), \quad\left(u_{1}, \cdots, u_{n}\right) \in[0,1]^{n},
$$

where $a_{i j}$ are real numbers fulfilling certain requirements so that $F\left(x_{1}, \cdots, x_{n}\right)$ is a proper $n$-dimensional distribution. For details of FGM distributions, see Kotz et al. [18].

It is easy to check that if the random variables $X_{1}, \cdots, X_{n}$ follow a joint $n$-dimensional FGM distribution defined in (2.4) and (2.5) whose marginal distributions $F_{k}(1 \leq k \leq n)$ are absolutely continuous and satisfy $F_{k}(-x)=o\left(\overline{F_{k}}(x)\right)$, then the four assumptions H1H4 are fulfilled.

\section{Results for finite sums}

In this section, we present our main results for finite sums.

Let $\left(X_{1}^{*}, \cdots, X_{n}^{*}\right)$ be an independent copy of $\left(X_{1}, \cdots, X_{n}\right)$, that is, $\left(X_{1}^{*}, \cdots, X_{n}^{*}\right)$ and $\left(X_{1}, \cdots, X_{n}\right)$ are two independent random vectors with the same marginal distributions and the components of $\left(X_{1}^{*}, \cdots, X_{n}^{*}\right)$ are independent. Similar to $S_{n}$ and $S_{n, k}$, we define $S_{n}^{*}=\sum_{k=1}^{n} X_{k}^{*}$, and $S_{n, k}^{*}=S_{n}^{*}-X_{k}^{*}$.

Theorem 3.1. Let $X_{1}, \cdots, X_{n}$ be heavy-tailed random variables with distribution $F_{1}, \cdots$, $F_{n}$, respectively. Under assumption $\mathbf{H 3}$,

(1) if $F_{1}, F_{2}, \cdots, F_{n} \in \mathcal{L}$, then

$$
\liminf _{x \rightarrow \infty} \frac{P\left(S_{n}>x\right)}{\sum_{k=1}^{n} \overline{F_{k}}(x)}=\liminf _{x \rightarrow \infty} \frac{P\left(S_{(n)}>x\right)}{\sum_{k=1}^{n} \overline{F_{k}}(x)}=1 ;
$$


(2) if additionally $P\left(\sum_{k=1}^{n} X_{k}^{*+}>x\right) \sim \sum_{k=1}^{n} \overline{F_{k}}(x)$, then

$$
\lim _{x \rightarrow \infty} \frac{P\left(S_{n}>x\right)}{\sum_{k=1}^{n} \overline{F_{k}}(x)}=\lim _{x \rightarrow \infty} \frac{P\left(S_{(n)}>x\right)}{\sum_{k=1}^{n} \overline{F_{k}}(x)}=1 .
$$

Proof. We first show that if $F_{1}, F_{2}, \cdots, F_{n} \in \mathcal{L}$, then

$$
\liminf _{x \rightarrow \infty} \frac{P\left(S_{n}>x\right)}{\sum_{k=1}^{n} \overline{F_{k}}(x)} \geq 1,
$$

under assumption $\mathbf{H} \mathbf{2}$.

It follows from the definition of the class $\mathcal{L}$ that there exists a sequence $\{a(x)\}$ such that $a(x) \rightarrow \infty$ as $x \rightarrow \infty, 2 a(x) \leq x$, and

$$
\bar{F}_{k}(x+a(x)) \sim \overline{F_{k}}(x), \quad x \rightarrow \infty, \quad k=1,2, \cdots, n .
$$

Note that

$$
\begin{aligned}
P\left(S_{n}>x\right) \geq & P\left(S_{n}>x, X_{(n)}>x+a(x)\right) \\
\geq & \sum_{k=1}^{n} P\left(S_{n}>x, X_{k}>x+a(x)\right) \\
& \quad-\sum_{1 \leq i<j \leq n} P\left(X_{i}>x+a(x), X_{j}>x+a(x)\right) \\
& \equiv I_{1}(x)+I_{2}(x) .
\end{aligned}
$$

Assumption $\mathbf{H 2}$ implies that

$$
I_{2}(x)=o\left(\sum_{k=1}^{n} \overline{F_{k}}(x)\right) .
$$

Write $S_{n, k}=S_{n}-X_{k}$ for $1 \leq k \leq n$. Then,

$$
\begin{aligned}
I_{1}(x) & \geq \sum_{k=1}^{n} P\left(S_{n, k}>-a(x), X_{k}>x+a(x)\right) \\
& =\sum_{k=1}^{n} P\left(X_{k}>x+a(x)\right)-\sum_{k=1}^{n} P\left(S_{n, k} \leq-a(x), X_{k}>x+a(x)\right) .
\end{aligned}
$$

It follows from (2.3) that

$$
\sum_{k=1}^{n} P\left(S_{n, k} \leq-a(x), X_{k}>x+a(x)\right)=o\left(\sum_{k=1}^{n} \overline{F_{k}}(x+a(x))\right) .
$$


Thus,

$$
\begin{aligned}
I_{1}(x) & \geq \sum_{k=1}^{n} P\left(X_{k}>x+a(x)\right)-o\left(\sum_{k=1}^{n} \overline{F_{k}}(x+a(x))\right) \\
& \sim \sum_{k=1}^{n} \overline{F_{k}}(x) .
\end{aligned}
$$

This proves (3.3). Note that (3.3) also holds under assumption H3 as H3 implies H2.

We next show that if $F_{1}, F_{2}, \cdots, F_{n} \in \mathcal{L}$ and assumption $\mathbf{H 3}$ holds, then

$$
\liminf _{x \rightarrow \infty} \frac{P\left(S_{n}>x\right)}{\sum_{k=1}^{n} \overline{F_{k}}(x)} \leq 1 .
$$

If additionally $P\left(\sum_{k=1}^{n} X_{k}^{*+}>x\right) \sim \sum_{k=1}^{n} \overline{F_{k}}(x)$, then

$$
\limsup _{x \rightarrow \infty} \frac{P\left(S_{n}>x\right)}{\sum_{k=1}^{n} \overline{F_{k}}(x)} \leq 1 .
$$

Assume that at least one of $X_{1}, \cdots, X_{n}$ has infinite mean. In this special case, (3.4) still holds without assumption H3. In fact, when $X_{1}, \cdots, X_{n}$ are nonnegative, one obtains

$$
\frac{\int_{0}^{t} P\left(S_{n}>x\right) d x}{\sum_{k=1}^{\infty} \int_{0}^{t} \overline{F_{k}}(x) d x}=\frac{E \min \left(S_{n}, t\right)}{\sum_{k=1}^{\infty} E \min \left(X_{k}, t\right)} \leq 1,
$$

for any positive $t$. This together with $\int_{0}^{\infty} \overline{F_{1}}(y) d y=\infty$ imply that

$$
\limsup _{x \rightarrow \infty} \frac{P\left(S_{n}>x\right)}{\sum_{k=1}^{n} \overline{F_{k}}(x)} \leq 1
$$

and hence

$$
\liminf _{x \rightarrow \infty} \frac{P\left(S_{n}>x\right)}{\sum_{k=1}^{n} \overline{F_{k}}(x)} \leq 1 .
$$

If at least one of $X_{1}^{+}=X_{1} 1\left(X_{1} \geq 0\right), \cdots, X_{n}^{+}=X_{n} 1\left(X_{n} \geq 0\right)$ has infinite mean, we may consider $X_{1}^{+}, \cdots, X_{n}^{+}$. If at least one of $X_{1}, \cdots, X_{n}$ has negative infinite mean, we may consider $-X_{1}, \cdots,-X_{n}$. So, in either case, we have upper bound (3.4).

Now, suppose that all random variables $X_{1}, \cdots, X_{n}$ have finite means. It is clear that the inequality

$$
P\left(X_{(n)}^{+}>x\right) \leq \sum_{k=1}^{n} \overline{F_{k}}(x) .
$$


holds. The conditions $F_{1}, F_{2}, \cdots, F_{n} \in \mathcal{L}$ and $\mathbf{H 3}$ imply that there exist positive constants $x_{0}$ and $d_{n}$ such that

$$
P\left(S_{n, k}>x \mid X_{k}=x_{x}\right) \leq d_{n} P\left(S_{n, k}^{*}>x\right)
$$

holds for all $1 \leq k \leq n, x>x_{0}$ and $x_{k}>x_{0}$; see Geluk and Tang [13]. It can be shown that for every function $a(\cdot):(0, \infty) \rightarrow\left(x_{0}, \infty\right)$ and for every $1 \leq k \leq n$, and $x>x_{0}$,

$$
P\left(S_{n}>x, a(x)<X_{k} \leq x\right) \leq d_{n} P\left(S_{n}^{*}>x, a(x)<X_{k}^{*} \leq x\right) .
$$

Using (1.1) and induction arguments, it follows from the proof of Lemma 5.2 of Geluk and Tang [13] that there exists a sequence $\left\{x_{l}\right\}$ such that $l \rightarrow \infty$ and

$$
P\left(S_{n}^{*}>x_{l}, \frac{x_{l}}{n}<X_{j}^{*} \leq x_{l}\right)=o\left(\sum_{k=1}^{n} \overline{F_{k}}\left(x_{l}\right)\right) .
$$

In particular, if $P\left(S_{n}^{*}>x\right) \sim \sum_{k=1}^{n} \overline{F_{k}}(x)$, then

$$
P\left(S_{n}^{*}>x, \frac{x}{n}<X_{j}^{*} \leq x\right)=o\left(\sum_{k=1}^{n} \overline{F_{k}}(x)\right) .
$$

Hence, (3.6), (3.7) and (3.8) imply that

$$
\begin{aligned}
P\left(S_{n}>x_{l}\right) & \leq P\left(\cup_{k=1}^{n}\left(X_{k}^{+}>x_{l}\right)\right)+P\left(S_{n}^{+}>x_{l}, \cap_{k=1}^{n}\left(X_{k}^{+} \leq x_{l}\right)\right) \\
& \leq P\left(X_{(n)}^{+}>x_{l}\right)+\sum_{k=1}^{n} P\left(S_{n}^{+}>x_{l}, \frac{x_{l}}{n}<X_{k}^{+} \leq x_{l}\right) \\
& \leq P\left(X_{(n)}^{+}>x_{l}\right)+d_{n} \sum_{k=1}^{n} P\left(S_{n}^{+*}>x_{l}, \frac{x_{l}}{n}<X_{k}^{+*} \leq x_{l}\right) \\
& \sim \sum_{k=1}^{n} \overline{F_{k}}\left(x_{l}\right) .
\end{aligned}
$$

This proves (3.4), and (3.5) can be proved using similar arguments.

Finally, (3.1) and (3.2) follow from (3.3), (3.4), (3.5) and the fact that

$$
P\left(\sum_{k=1}^{n} X_{k}^{+}>x\right) \geq P\left(S_{(n)}>x\right) \geq P\left(S_{n}>x\right) .
$$

This completes the proof of Theorem 3.1.

Remark 3.1. Let $X_{1}, \cdots, X_{n}$ be $n$ nonnegative heavy-tailed random variables. It follows from Foss and Korshunov [11] that there exist two sequences $\left\{x_{l}\right\}$ and $\left\{a\left(x_{l}\right)\right\}$ such that $x_{l} \rightarrow \infty, a\left(x_{l}\right) \rightarrow \infty$ as $l \rightarrow \infty, 2 a\left(x_{l}\right) \leq x_{l}$, and

$$
\bar{F}_{k}\left(x_{l}+a\left(x_{l}\right)\right) \sim \overline{F_{k}}\left(x_{l}\right), \quad l \rightarrow \infty, \quad k=1,2, \cdots, n .
$$


Repeating the same arguments as above we find that (3.3) also holds without the assumption that $F_{1}, F_{2}, \cdots, F_{n} \in \mathcal{L}$.

Note that for $n=2$ and nonnegative random variables, a result similar to (3.2) was established by Foss and Korshunov [11] under the independence assumption.

Corollary 3.1. Let $X_{1}, \cdots, X_{n}$ be heavy-tailed random variables with common distribution $F$. Under assumption $\mathbf{H 3}$, if $F \in \mathcal{L}$, then

$$
\liminf _{x \rightarrow \infty} \frac{P\left(S_{n}>x\right)}{\bar{F}(x)}=\liminf _{x \rightarrow \infty} \frac{P\left(S_{(n)}>x\right)}{\bar{F}(x)}=n ;
$$

and if $F \in \mathcal{S}$, then

$$
\lim _{x \rightarrow \infty} \frac{P\left(S_{n}>x\right)}{\bar{F}(x)}=\lim _{x \rightarrow \infty} \frac{P\left(S_{(n)}>x\right)}{\bar{F}(x)}=n
$$

If we switch our attention from the class $\mathcal{L}$ to the class $\mathcal{D}$, we only need assumption $\mathbf{H} \mathbf{2}$ which is weaker than $\mathbf{H 3}$.

Theorem 3.2. Let $X_{1}, \cdots, X_{n}$ be $n$ nonnegative heavy-tailed random variables with distribution $F_{1}, \cdots, F_{n}$, respectively. Under assumption $\mathbf{H 2}$, if $F_{k} \in \mathcal{D}$ for $k=1, \cdots, n$, then

$$
\liminf _{x \rightarrow \infty} \frac{P\left(S_{n}>x\right)}{\sum_{k=1}^{n} \overline{F_{k}}(x)}=\liminf _{x \rightarrow \infty} \frac{P\left(S_{(n)}>x\right)}{\sum_{k=1}^{n} \overline{F_{k}}(x)}=1 ;
$$

Moreover, if $F_{k} \in \mathcal{L}$ for $k=1, \cdots, n$, then

$$
\lim _{x \rightarrow \infty} \frac{P\left(S_{n}>x\right)}{\sum_{k=1}^{n} \overline{F_{k}}(x)}=\lim _{x \rightarrow \infty} \frac{P\left(S_{(n)}>x\right)}{\sum_{k=1}^{n} \overline{F_{k}}(x)}=1 .
$$

Proof. It follows from Foss and Korshunov [11] that there exist two sequences $\left\{x_{l}\right\}$ and $\left\{a\left(x_{l}\right)\right\}$ such that $x_{l} \rightarrow \infty, a\left(x_{l}\right) \rightarrow \infty$ as $l \rightarrow \infty, 2 a\left(x_{l}\right) \leq x_{l}$, and

$$
\bar{F}_{k}\left(x_{l}-a\left(x_{l}\right)\right) \sim \overline{F_{k}}\left(x_{l}\right), k=1,2, \cdots, n .
$$

Note that

$$
\begin{aligned}
P\left(S_{n}>x_{l}\right) & \leq P\left(S_{n}>x_{l}, X_{(n)}>x_{l}-a\left(x_{l}\right)\right)+P\left(S_{n}>x_{l}, \cap_{k=1}^{n}\left(X_{k} \leq x_{l}-a\left(x_{l}\right)\right)\right. \\
& \leq \sum_{k=1}^{n} P\left(X_{k}>x_{l}-a\left(x_{l}\right)\right)+\sum_{k=1}^{n} P\left(S_{n}-X_{k}>a\left(x_{l}\right), X_{k}>\frac{x_{l}}{n}\right) \\
& \equiv J_{1}\left(x_{l}\right)+J_{2}\left(x_{l}\right) .
\end{aligned}
$$


It is obvious that $J_{1}\left(x_{l}\right) \sim \sum_{k=1}^{n} \overline{F_{k}}\left(x_{l}\right)$. Recall that $S_{n, k}=S_{n}-X_{k}$ for $1 \leq k \leq n$. Under assumption $\mathbf{H 2}$, we have

$$
\begin{aligned}
J_{2}\left(x_{l}\right) & =\sum_{k=1}^{n} P\left(S_{n, k}>a\left(x_{l}\right), X_{k}>\frac{x_{l}}{n}\right) \\
& \leq \sum_{1 \leq k \neq j \leq n} P\left(X_{j}>\frac{a\left(x_{l}\right)}{n-1}, X_{k}>\frac{x_{l}}{n}\right) \\
& \leq \sum_{1 \leq k \neq j \leq n} P\left(X_{j}>\frac{a\left(x_{l}\right)}{n-1} \mid X_{k}>\frac{x_{l}}{n}\right) P\left(X_{k}>\frac{x_{l}}{n}\right) \\
& =o(1) \sum_{k=1}^{n} \overline{F_{k}}\left(x_{l}\right),
\end{aligned}
$$

where, in the last step, we used the relation $\bar{F}_{k}(x y)=O\left(\bar{F}_{k}(x)\right)$ for all $y>0\left(\right.$ as $\left.F_{k} \in \mathcal{D}\right)$. Thus, we obtain

$$
\liminf _{x \rightarrow \infty} \frac{P\left(S_{n}>x\right)}{\sum_{k=1}^{n} \overline{F_{k}}(x)} \leq 1
$$

This together with (3.3) (see Remark 3.1) imply that

$$
\liminf _{x \rightarrow \infty} \frac{P\left(S_{n}>x\right)}{\sum_{k=1}^{n} \overline{F_{k}}(x)}=1
$$

Furthermore, if $F_{k} \in \mathcal{L}$ for $k=1, \cdots, n$, then (3.13) holds for any $x$. Repeating the steps above, we obtain

$$
\limsup _{x \rightarrow \infty} \frac{P\left(S_{n}>x\right)}{\sum_{k=1}^{n} \overline{F_{k}}(x)} \leq 1 .
$$

The remaining proofs of (3.11) and (3.12) are straightforward.

The last result in this section is trivial.

Theorem 3.3. Let $X_{1}, \cdots, X_{n}$ be heavy-tailed random variables with distribution $F_{1}, \cdots$, $F_{n}$, respectively. Under assumption $\mathbf{H} \mathbf{1}$, we have

$$
\lim _{x \rightarrow \infty} \frac{P\left(X_{(n)}>x\right)}{\sum_{k=1}^{n} \overline{F_{k}}(x)}=1 .
$$

Proof. On one hand, the inequality $P\left(X_{(n)}>x\right) \leq \sum_{k=1}^{n} \overline{F_{k}}(x)$ is trivial. On the other hand,

$$
P\left(X_{(n)}>x\right) \geq \sum_{k=1}^{n} P\left(X_{k}>x\right)-\sum_{1 \leq i \neq j \leq n} P\left(X_{i}>x, X_{j}>x\right) .
$$


Under assumption $\mathbf{H 1}$

$$
\sum_{1 \leq i \neq j \leq n} P\left(X_{i}>x, X_{j}>x\right) \leq o(1) \sum_{k=1}^{n} \overline{F_{k}}(x) .
$$

The result of Theorem 3.3 follows from the above inequalities.

Remark 3.2. For heavy-tailed random variables $X_{1}, \cdots, X_{n}$ with distribution $F_{1}, \cdots$, $F_{n}$, if $F_{1}, F_{2}, \cdots, F_{n} \in \mathcal{S}, F_{i} * F_{j} \in \mathcal{S}$ for all $1 \leq i \neq j \leq n$, and assumption H2 holds, then the result of Geluk and Tang ([13, Theorem 3.2]) gives

$$
\lim _{x \rightarrow \infty} \frac{P\left(S_{n}>x\right)}{\sum_{k=1}^{n} \overline{F_{k}}(x)}=1 .
$$

Obviously, the conditions in Theorem 3.1 are slightly more general than the above conditions of Geluk and Tang ([13, Theorem 3.2]). Furthermore, (3.10) proves the insensitivity of relation $P\left(S_{n}>x\right) \sim P\left(S_{(n)}>x\right) \sim n \bar{F}(x)$ to the dependence assumption in H2, and Theorem 3.3 proves the insensitivity of relation $P\left(X_{(n)}>x\right) \sim n \bar{F}(x)$ to the dependence assumption in $\mathbf{H} \mathbf{1}$.

\section{Results for random sums}

Parallel to the result of Denisov et al. [6] for i.i.d. random variables with a support on $[0, \infty)$, we obtain the following theorem for heavy-tailed random variables satisfying some dependence structure.

Theorem 4.1. Let $X_{1}, \cdots, X_{n}$ be heavy-tailed random variables with common distribution $F$, and $\tau$ be a counting random variable independent of the sequence $\left\{X_{k}\right\}$. Assume that $E\left(z^{\tau}\right)<\infty$ for some $z>1$. Under assumption $\mathbf{H 3}$, if $F \in \mathcal{L}$, then

$$
\liminf _{x \rightarrow \infty} \frac{P\left(S_{\tau}>x\right)}{\bar{F}(x)}=E \tau
$$

and if $F \in \mathcal{S}$, then

$$
\lim _{x \rightarrow \infty} \frac{P\left(S_{\tau}>x\right)}{\bar{F}(x)}=E \tau
$$

Proof. Note that the condition on $E\left(z^{\tau}\right)<\infty$ implies the existence of $E \tau$ and $E(1+\varepsilon)^{\tau}<$ $\infty$ for some sufficiently small $\varepsilon>0$. For such $\varepsilon$, it follows from (3.9) that there exist a 
constant $K \equiv K(\varepsilon)<\infty$ and a sequence $\left\{x_{l}\right\}$ such that for all $n \geq 2$

$$
P\left(S_{n}>x_{l}\right) \sim n \bar{F}\left(x_{l}\right), \quad P\left(S_{n}>x_{l}\right) \leq K(1+\varepsilon)^{n} \bar{F}\left(x_{l}\right), \quad l \geq 1 .
$$

Applying the dominated convergence theorem yields

$$
\lim _{l \rightarrow \infty} \frac{P\left(S_{\tau}>x_{l}\right)}{\bar{F}\left(x_{l}\right)}=\lim _{l \rightarrow \infty} \sum_{n=1}^{\infty} P(\tau=n) \frac{P\left(S_{n}>x_{l}\right)}{\bar{F}\left(x_{l}\right)}=E \tau,
$$

which implies (4.1). Furthermore, if $F \in \mathcal{S}$, then (4.2) follows from (3.10) easily.

Here, we also extend the results of Denisov et al. [7] to the case of dependent heavytailed random variables. Note that their asymptotic results are for sums of random size $\tau$ of i.i.d. nonnegative heavy-tailed random variables with $\tau$ belonging to the class of all light-tailed distributions and also to some class of heavy-tailed distributions.

Theorem 4.2. Let $X_{1}, \cdots, X_{n}$ be heavy-tailed random variables with common distribution $F$, and $\tau$ be a counting random variable independent of the sequence $\left\{X_{k}\right\}$ with infinite mean. Under assumption $\mathbf{H 2}$, we have

$$
\liminf _{x \rightarrow \infty} \frac{P\left(S_{\tau}>x\right)}{\bar{F}(x)}=\liminf _{x \rightarrow \infty} \frac{P\left(S_{(\tau)}>x\right)}{\bar{F}(x)}=\lim _{x \rightarrow \infty} \frac{P\left(X_{(\tau)}>x\right)}{\bar{F}(x)}=E \tau .
$$

Proof. Since $\tau$ is independent of $X_{k}$ 's, we can write

$$
P\left(S_{\tau}>x\right)=\sum_{n=0}^{\infty} P(\tau=n) P\left(S_{n}>x\right) .
$$

Using (3.3) and Fatou's lemma, we obtain

$$
\liminf _{x \rightarrow \infty} \frac{P\left(S_{\tau}>x\right)}{\bar{F}(x)} \geq \sum_{n=0}^{\infty} n P(\tau=n)=E \tau .
$$

We immediately get the first equality in (4.3) since $E \tau=\infty$. The rest of the proof is similar.

The next two theorems consider the case with $E \tau<\infty$.

Theorem 4.3. Let $X_{1}, \cdots, X_{n}$ be heavy-tailed random variables with common distribution $F$, and $\tau$ be a counting random variable independent of the sequence $\left\{X_{k}\right\}$. Assume that $E \tau<\infty$. Under assumption $\mathbf{H 1}$, we have

$$
\lim _{x \rightarrow \infty} \frac{P\left(X_{(\tau)}>x\right)}{\bar{F}(x)}=E \tau .
$$


Proof. The proof is straightforward.

Theorem 4.4. Let $X_{1}, \cdots, X_{n}$ be heavy-tailed random variables with common distribution $F$ and finite mean, and $\tau$ be a counting random variable independent of the sequence $\left\{X_{k}\right\}$ with finite mean $E \tau$. Under assumption $\mathbf{H 4}$, we have the following results:

(i) Assume that $E X_{1}<0$. If $F \in \mathcal{S}$, then

$$
\liminf _{x \rightarrow \infty} \frac{P\left(S_{\tau}>x\right)}{\bar{F}(x)}=\liminf _{x \rightarrow \infty} \frac{P\left(S_{(\tau)}>x\right)}{\bar{F}(x)}=E \tau .
$$

Moreover, if $F \in \mathcal{S}_{*}$, then

$$
\lim _{x \rightarrow \infty} \frac{P\left(S_{\tau}>x\right)}{\bar{F}(x)}=\lim _{x \rightarrow \infty} \frac{P\left(S_{(\tau)}>x\right)}{\bar{F}(x)}=E \tau .
$$

(ii) Assume that $E X_{1} \geq 0$ and that there exists $c>E \xi_{1}$ such that $P(c \tau>x)=o(\bar{F}(x))$ as $x \rightarrow \infty$. If $F \in \mathcal{S}$, then

$$
\liminf _{x \rightarrow \infty} \frac{P\left(S_{\tau}>x\right)}{\bar{F}(x)}=\liminf _{x \rightarrow \infty} \frac{P\left(S_{(\tau)}>x\right)}{\bar{F}(x)}=E \tau .
$$

Moreover, if $F \in \mathcal{S}^{*}$, then

$$
\lim _{x \rightarrow \infty} \frac{P\left(S_{\tau}>x\right)}{\bar{F}(x)}=\lim _{x \rightarrow \infty} \frac{P\left(S_{(\tau)}>x\right)}{\bar{F}(x)}=E \tau .
$$

Proof. We first prove (i). Since $F \in \mathcal{S}$ is heavy tailed and has a finite mean, it follows from Lemma 4 of Foss and Korshunov [11] that there exists a sequence $\left\{x_{l}\right\}$ such that $x_{l} \rightarrow \infty$ as $l \rightarrow \infty$ and

$$
\lim _{l \rightarrow \infty} \frac{1}{\bar{F}\left(x_{l}\right)} \int_{0}^{x_{l}} \bar{F}\left(x_{l}-y\right) \bar{F}(y) d y=2 \int_{0}^{\infty} \bar{F}(z) d z .
$$

Lemma 9 of Denisov et al. [5] implies that $\overline{F_{h} * F_{h}}\left(x_{l}\right) \sim 2 \overline{F_{h}}\left(x_{l}\right)$ uniformly in $h \in[1, \infty)$ as $l \rightarrow \infty$, where $F_{h}$ is defined in (2.1). If $E X_{1}<0$, it follows from the result of Korshunov [19] that

$$
P\left(S_{(n)}^{*}>x_{l}\right) \sim \frac{1}{\left|E X_{1}\right|} \int_{x_{l}}^{x_{l}+n\left|E X_{1}\right|} \bar{F}(y) d y,
$$

uniformly in $n \geq 1$. Consider the relation

$$
P\left(S_{(\tau)}>x_{l}\right) \sim E \tau \bar{F}\left(x_{l}\right) .
$$


Since $c\left(u_{1}, \cdots, u_{n}\right)<M$ for all $\left(u_{1}, \cdots, u_{n}\right) \in[0,1]^{n}$, then for any $x$,

$$
P\left(S_{(n)}>x\right) \leq M P\left(S_{(n)}^{*}>x\right)
$$

Thus, from (4.10), we have

$$
P\left(S_{(n)}>x_{l}\right) \leq M(1+o(1)) n\left|E X_{1}\right| \bar{F}\left(x_{l}\right)
$$

for all $n \geq 1$. Applying the dominated convergence theorem and (3.10), we obtain

$$
\lim _{l \rightarrow \infty} \frac{P\left(S_{(\tau)}>x_{l}\right)}{\bar{F}\left(x_{l}\right)}=\sum_{k=1}^{\infty}\left(\lim _{l \rightarrow \infty} \frac{P\left(S_{(n)}>x_{l}\right)}{\bar{F}\left(x_{l}\right)}\right) P(\tau=n)=E \tau,
$$

which proves (4.11). Furthermore, Fatou's lemma gives

$$
\liminf _{x \rightarrow \infty} \frac{P\left(S_{\tau}>x\right)}{\bar{F}(x)} \geq E \tau
$$

without any restriction on the sign of $E X_{1}$. Since $P\left(S_{\tau}>x\right) \leq P\left(S_{(\tau)}>x\right)$ for all $x$, (4.5) follows from (4.13) and (4.14). On the other hand, if $F \in \mathcal{S}_{*}$ and $E X_{1}<0$, then (4.10) holds for any $x$, so that (4.6) can be proved by modifying the proof of (4.5).

To prove (ii), it is sufficient to prove (4.11) for some sequence $\left\{x_{l}\right\}$. Since $F \in \mathcal{S}$, it follows from (3.10) that

$$
P\left(S_{(n)}>x\right) \sim n \bar{F}(x) .
$$

Thus, there exists an increasing function $N(x) \rightarrow \infty$ such that

$$
P_{1}(x):=P\left(S_{(\tau)}>x, \tau \leq N(x)\right) \sim E \tau \bar{F}(x) .
$$

Let $\varepsilon=\left(c-E X_{1}\right) / 2>0$ and $b=\left(E X_{1}+c\right) / 2$. Put $\tilde{X}_{i}=X_{i}-b$ and $\tilde{S}_{n}=\tilde{X}_{1}+\cdots+\tilde{X}_{n}$. Then, $E \tilde{X}_{i}=-\varepsilon<0$. Using (4.10) and (4.12), we have

$$
P\left(S_{(n)}>x_{l}\right) \leq M P\left(S_{(n)}^{*}>x_{l}\right) \leq M P\left(\tilde{S}_{(n)}^{*}>x_{l}-b n\right)
$$

Following the steps of the proof of Theorem 1 (ii) of Denisov et al. [8], one gets

$$
P_{2}\left(x_{l}\right):=P\left(S_{(\tau)}>x_{l}, \tau \in\left(N\left(x_{l}\right), x_{l} / c\right]\right)=o\left(\bar{F}\left(x_{l}\right)\right) .
$$

Finally, the condition $P(c \tau>x)=o(\bar{F}(x))$ gives

$$
P_{3}(x):=P\left(S_{(\tau)}>x, c \tau>x\right)=o(\bar{F}(x)) .
$$


Thus,

$$
P\left(S_{(\tau)}>x_{l}\right) \equiv P_{1}\left(x_{l}\right)+P_{2}\left(x_{l}\right)+P_{3}\left(x_{l}\right) \sim E \tau \bar{F}\left(x_{l}\right) \text { as } l \rightarrow \infty .
$$

Also, if $F \in \mathcal{S}^{*}$, then

$$
P\left(S_{(\tau)}>x\right) \equiv P_{1}(x)+P_{2}(x)+P_{3}(x) \sim E \tau \bar{F}(x), \text { as } x \rightarrow \infty .
$$

Hence, the proof of (ii) is complete.

Remark 4.1. Theorem 4.4 partially extends (3.9) and (3.10) to the case of random sums. Under the independence assumption and for $F \in \mathcal{S}^{*}$, (4.6) was established in Denisov et al. ([8, Theorem 1(i)]). Here, (4.8) generalizes the result of Denisov et al. ([8, Theorem 1(ii)]) to the dependent case. For related work, we refer the reader to $\mathrm{Ng}$ et al. [22], and $\mathrm{Ng}$ and Tang [21]. Concerning the asymptotics for the maximum of $S_{(\tau)}$, it was shown in Foss and Zachary [12] that the relation $P\left(S_{(\tau)}>x\right) \sim E \tau \bar{F}(x)$ holds for any stopping time $\tau \leq \infty$ and $F \in \mathcal{S}_{*}$.

\section{Applications to risk theory}

In this section, we present an example to illustrate some applications of our main results.

Example 5.1. Following the formulation of $\mathrm{Ng}$ et al. [20], we can write the surplus of an insurance company at time $t$ as

$$
U_{\delta}(t)=x e^{\delta t}-\sum_{k=1}^{N(t)} X_{k} e^{\delta\left(t-\sigma_{k}\right)}, t \geq 0,
$$

where $x \geq 0$ is the initial surplus, $\delta \geq 0$ is the constant interest force, $\sigma_{k}$ is the time at which the $k$ th customer arrives and buys an insurance contract with $\sigma_{0}=0, N(t)=$ $\max \left\{k \geq 0: \sigma_{k} \leq t\right\}$ is the individual customer-arrival process, and $X_{k}$ represents the total potential claims due to the $k$ th customer. In this example, $\left\{X_{k}, k \geq 1\right\}$ is a sequence of random variables which are not necessarily i.i.d. Furthermore, if $\sigma_{k}=k$ for each $k \geq 1$, then risk process (5.1) can be rewritten as

$$
U_{\delta}(0)=x, \quad U_{\delta}(n)=x(1+r)^{n}-\sum_{k=1}^{n} X_{k}(1+r)^{n-k}, \quad n=1,2 . \cdots,
$$


where $r=e^{\delta}-1$. In the literature, model (5.2) corresponds to a discrete-time insurance risk model with a constant interest rate (see, for example, Tang [24]).

Let $V_{k}=X_{k}(1+r)^{-k}$ and $S_{n}=\sum_{k=1}^{n} V_{k}$. Then, we can rewrite (5.2) as $U_{\delta}(n)=$ $(1+r)^{n}\left(x-S_{n}\right)$. Define $n$-period finite-time ruin probability as

$$
\psi_{n}(x)=P\left(\inf _{1 \leq k \leq n} U_{\delta}(k)<0 \mid U_{\delta}(0)=x\right)=P\left(\max _{1 \leq k \leq n} S_{k}>x\right) .
$$

From Theorem 3.1, we obtain

Corollary 5.1. Assume that $X_{k} \sim F_{k}$ and hence $V_{k}$ are heavy-tailed random variables for $k=1,2, \cdots$. Under assumption $\mathbf{H 3}$, if $F_{1}, F_{2}, \cdots, F_{n} \in \mathcal{L}$, then

$$
\liminf _{x \rightarrow \infty} \frac{\psi_{n}(x)}{\sum_{k=1}^{n} \overline{F_{k}}\left(x(1+r)^{k}\right)}=1 \text {; }
$$

and if additionally $P\left(\sum_{k=1}^{n} V_{k}^{*+}>x\right) \sim \sum_{k=1}^{n} \overline{F_{k}}(x)$, then

$$
\lim _{x \rightarrow \infty} \frac{\psi_{n}(x)}{\sum_{k=1}^{n} \overline{F_{k}}\left(x(1+r)^{k}\right)}=1 \text {. }
$$

Note that if $\delta=0$ and $X_{k}=Z_{k}-(1+\rho) \mu$, where $\left\{Z_{k}, k \geq 1\right\}$ is a sequence of nonnegative random variables with common distribution function $\mathrm{F}$ and finite mean $\mu$, and the positive constant $\rho$ can be interpreted as the safety loading, then (5.1) has the form

$$
U_{\delta}(t)=x-\sum_{k=1}^{N(t)} X_{k} \equiv x-S_{N(t)} .
$$

which is the so-called customer-arrival-based insurance risk model studied by $\mathrm{Ng}$ et al. $[20]$.

For the ruin probability within a finite horizon $T$ given by

$$
\psi(x ; T)=P\left(\inf _{0 \leq t \leq T} U(t)<0\right)=P\left(S_{(N(T))}>x\right),
$$

one can apply Theorem 4.4 to obtain

Corollary 5.2. Let $\left\{Z_{k}, k \geq 1\right\}$ independent of $N(t)$ be a sequence of heavy-tailed random variables with common distribution $F$. Under the conditions in Theorem 4.4, if $F \in \mathcal{S}$, then

$$
\liminf _{x \rightarrow \infty} \frac{\psi(x ; T)}{\bar{F}(x)}=\lambda(T) .
$$


Also, if $F \in \mathcal{S}^{*}$, then

$$
\lim _{x \rightarrow \infty} \frac{\psi(x ; T)}{\bar{F}(x)}=\lambda(T)
$$

Remark 5.1. Under the independent setting of $\left\{Z_{k}, k \geq 1\right\}$ and other conditions, (5.3) were obtained in several papers. For example, $\mathrm{Ng}$ et al. [22] obtained (5.3) under the conditions that $F \in \mathcal{L} \cap \mathcal{D}$ and $P(N(T)>x)=o(\bar{F}(x))$; Ng et al. [20] obtained (5.3) under the conditions that $\left\{Z_{k}, k \geq 1\right\}$ is a sequence of i.i.d. subexponential random variables and $E(1+\varepsilon)^{N(T)}<\infty$ for some $\varepsilon>0$; and Kass and Tang [14] weakened the condition on $N(\cdot)$ and obtained (5.3) under the condition that $F$ is strongly subexponential, that is, $F \in \mathcal{S}_{*}$.

Acknowledgements. The authors are thankful to Professor Qihe Tang for his helpful comments and constructive suggestions, which have considerably enhanced this work. The research of Kam C. Yuen was supported by a university research grant of the University of Hong Kong. The research of Chuancun Yin was supported by the National Natural Science Foundation of China (No. 10771119) and the Research Fund for the Doctoral Program of Higher Education of China (No. 20093705110002).

\section{References}

[1] Albrecher, H., Asmussen, S. and Kortschak, D. (2006). Tail asymptotics for the sum of two heavy-tailed dependent risks. Extremes 9, 107-130.

[2] Asmussen, S. (2000). Ruin Probabilities. World Scientific, Singapore.

[3] Bingham, N.H., Goldie, C.M. and Teugels, J.L. (1987). Regular Variation. Cambridge University Press, Cambridge.

[4] Chen, Y. and Yuen, K.C. (2009). Sums of pairwise quasi-asymptotically independent random variables with consistent variation. Stoch. Models 25, 76-89.

[5] Denisov, D., Foss, S. and Korshunov, D. (2004). Tail asymptotics for the supremum of a random walk when the mean is not finite. Queueing Syst. 46, 15-33. 
[6] Denisov, D., Foss, S. and Korshunov, D. (2008). On lower limits and equivalences for distribution tails of randomly stopped sums. Bernoulli 14, 391-404.

[7] Denisov, D., Foss, S. and Korshunov, D. (2008). Lower limits for distribution tails of randomly stopped sums. Theory Probab. Appl. 52, 690-699.

[8] Denisov, D., Foss, S. and Korshunov, D. (2010). Asymptotics of randomly stopped sums in the presence of heavy tails. Bernoulli (in press).

[9] Embrechts, P., Goldie, C.M. and Veraverbeke, N. (1979). Subexponentiality and infinite divisibility. Z. Wahrsch. verw. Gebiete 49, 335-347.

[10] Embrechts, P., Klüppelberg, C. and Mikosch, T. (1997). Modelling Extremal Events for Insurance and Finance. Springer, Berlin.

[11] Foss, S. and Korshunov, D. (2007). Lower limits and equivalences for convolution tails. Ann. Probab. 35, 366-383.

[12] Foss, S. and Zachary, S. (2003). The maximum on a random time interval of a random walk with long-tailed increments and negative drift. Ann. Appl. Probab. 13, 37-53.

[13] Geluk, J. and Tang, Q.H. (2009). Asymptotic tail probabilities of sums of dependent subexponential random variables: J. Theoret. Probab. 22, 871-882.

[14] Kaas, R. and Tang, Q.H. (2003). Note on the tail behavior of random walk maxima with heavy tails and negative drift. North Amer. Actuar. J. 7(3), 57-61.

[15] Klüppelberg, C. (1988). Subexponential distributions and integrated tails. J. Appl. Probab. 25, 132-141.

[16] Ko, B. and Tang, Q.H. (2008). Sums of dependent nonnegative random variables with subexponential tails. J. Appl. Probab. 45, 85-95.

[17] Korshunov, D. (2002). Large-deviation probabilities for maxima of sums of independent random variables with negative mean and subexponential distribution. Theory Probab. Appl. 46, 355-366.

[18] Kotz, S., Balakrishnan, N. and Johnson, N.L. (2000).Continuous Multivariate Distributions: Models and Applications (Vol. 1, Second edition). Wiley, New York. 
[19] Nelsen, R.B. (2006). An Introduction to Copulas (Second edition). Springer, New York.

[20] Ng, K.W., Tang, Q.H., Yan, J.A. and Yang, H.L. (2003). Precise large deviations for the prospective-loss process. J. Appl. Probab. 40, 391-400.

[21] Ng, K.W. and Tang, Q.H. (2004). Asymptotic behavior of tail and local probabilities for sums of subexponential random variables. J. Appl. Probab. 41, 108-116.

[22] Ng, K.W., Tang, Q. H. and Yang, H.L. (2002). Maxima of sums of heavy-tailed random variables. ASTIN Bull. 32, 43-55.

[23] Resnick, S.I. (2002). Hidden regular variation, second order regular variation and asymptotic independence. Extremes 5, 303-336.

[24] Tang, Q.H. (2004). The ruin probability of a discrete time risk model under constant interest rate with heavy tails.Scandinavian Actuarial Journal 3, 229-240.

[25] Yu, C., Wang, Y. and Cui, Z. (2010). Lower limits and upper limits for tails of random sums supported on $\mathbb{R}$. Statist. Prob. Lett. preprint. 